\title{
Experimental research on the effect of microRNA-21 inhibitor on a rat model of intervertebral disc degeneration
}

\author{
XIAOMING SHENG ${ }^{1}$, QINGSONG GUO ${ }^{2}$, JUNBO YU $^{3}$ and YOUJIA XU ${ }^{1}$ \\ ${ }^{1}$ Department of Orthopedics, The Affiliated Second Hospital of Soochow University, Suzhou, Jiangsu 215000; \\ Departments of ${ }^{2}$ General Surgery and ${ }^{3}$ Emergency, The First Affiliated Hospital of Nantong University, \\ Nantong, Jiangsu 225001, P.R. China
}

Received August 28, 2017; Accepted January 8, 2018

DOI: $10.3892 /$ etm.2018.6156

\begin{abstract}
Intervertebral disc degeneration is associated with angiogenesis and is the primary cause of disc-associated disease. Several studies have indicated the importance of microRNA (miR)-21 in angiogenesis. Thus, the present study aimed to validate the role and underlying mechanisms of miR-21 in a rat model of intervertebral disc degeneration. A total of 60 specific-pathogen-free Sprague-Dawley rats were used for in vivo experiments. A rat model of intervertebral disc degeneration was established and miR-21 inhibitor (antagomiR-21) was administered. The vertebral pulp and annulus fibrosus were isolated for immunohistochemical analysis of hypoxia inducible factor (HIF)- $1 \alpha$ and vascular endothelial growth factor (VEGF) expression. Lumbar spine proteoglycan content was detected with the phloroglucinol method. Disc cell apoptosis was detected with terminal deoxynucleotidyl-transferase-mediated dUTP nick end labeling staining. It was revealed that antagomiR-21 treatment decreased the expression of HIF- $1 \alpha$ and VEGF in the vertebral pulp and annulus fibrosus. Furthermore, antagomiR-21 treatment increased proteoglycan content and inhibited cell apoptosis in lumbar spines from model rats with intervertebral disc degeneration. In conclusion, antagomiR-21 treatment exerted a protective role in a rat model of intervertebral disc degeneration, which may provide the basis for a potential therapeutic approach in the treatment of disc-associated diseases.
\end{abstract}

\section{Introduction}

Intervertebral disc degeneration is a major pathological process that occurs in the lower back and is the main cause of disc-associated diseases, including disc herniation and spinal stenosis (1-4). Previous studies have indicated that the

Correspondence to: Dr Youjia Xu, Department of Orthopedics, The Affiliated Second Hospital of Soochow University, 1055 Sanxiang Road, Suzhou, Jiangsu 215000, P.R. China

E-mail: xuyoujia@medmail.com.cn

Key words: microRNA-21, hypoxia inducible factor-1 $1 \alpha$, vascular endothelial growth factor, intervertebral disc degeneration underlying cause of disc degeneration is tissue weakening, which occurs primarily due to genetic inheritance, aging, inadequate nutritional status and loading history (5). Several other factors may also influence the aging and degeneration of discs, including metabolite transport impairment, cell senescence and death, genetic inheritance, changes in matrix macromolecules and water content, alterations in enzyme activity, structural failure and neurovascular ingrowth (5).

Several studies have reported the important role of angiogenesis in degeneration of the intervertebral disc (6,7). Degenerative intervertebral disc disorders are thought to be characterized by angiogenesis and the increased expression of vascular endothelial growth factor (VEGF), an angiogenic factor (8). Wang et al (9) also concluded that degeneration of the intervertebral disc was accompanied by angiogenesis. David et al (10) demonstrated that angiogenesis influences the pain intensity of intervertebral disc hernias and negatively impacts postoperative pain improvement, mobility and overall quality of life.

MicroRNAs (miRNAs/miRs) are small non-coding RNAs composed of 20-22 nucleotides, which inhibit protein expression by binding to the 3'-untranslated region of target mRNAs, leading to transcriptional repression or degradation of the mRNA (11). MiR-21 is an oncogenic miRNA that is overexpressed in several human tumors and has the ability to modulate cancer-associated target gene expression (12). Notably, it has been reported that miR-21 overexpression impairs angiogenesis in normal epithelial cells (13). Zhao et al (12) demonstrated that arsenite-induced carcinogenesis involves angiogenesis mediated by miR-21. Liu et al (14) revealed that miR-21 overexpression induced tumor angiogenesis and increased the expression of hypoxia inducible factor (HIF). HIF-1 $\alpha$ has an important role in angiogenesis and can stimulate the expression of VEGF (15); HIF-1 $\alpha$ has also been reported to play an important role in the development of degenerative processes in the intervertebral discs of mice (16).

The aforementioned studies indicate the importance of miR-21 in angiogenesis. The present study aimed to investigate if miR-21 had a critical role in the progression of intervertebral disc degeneration through HIF-1 $\alpha$ and VEGF expression regulation. A rat model of intervertebral disc degeneration was established and the model rats were administered miR-21 inhibitor (antagomiR-21). The vertebral pulp and annulus fibrosus were isolated for immunohistochemical analysis to 

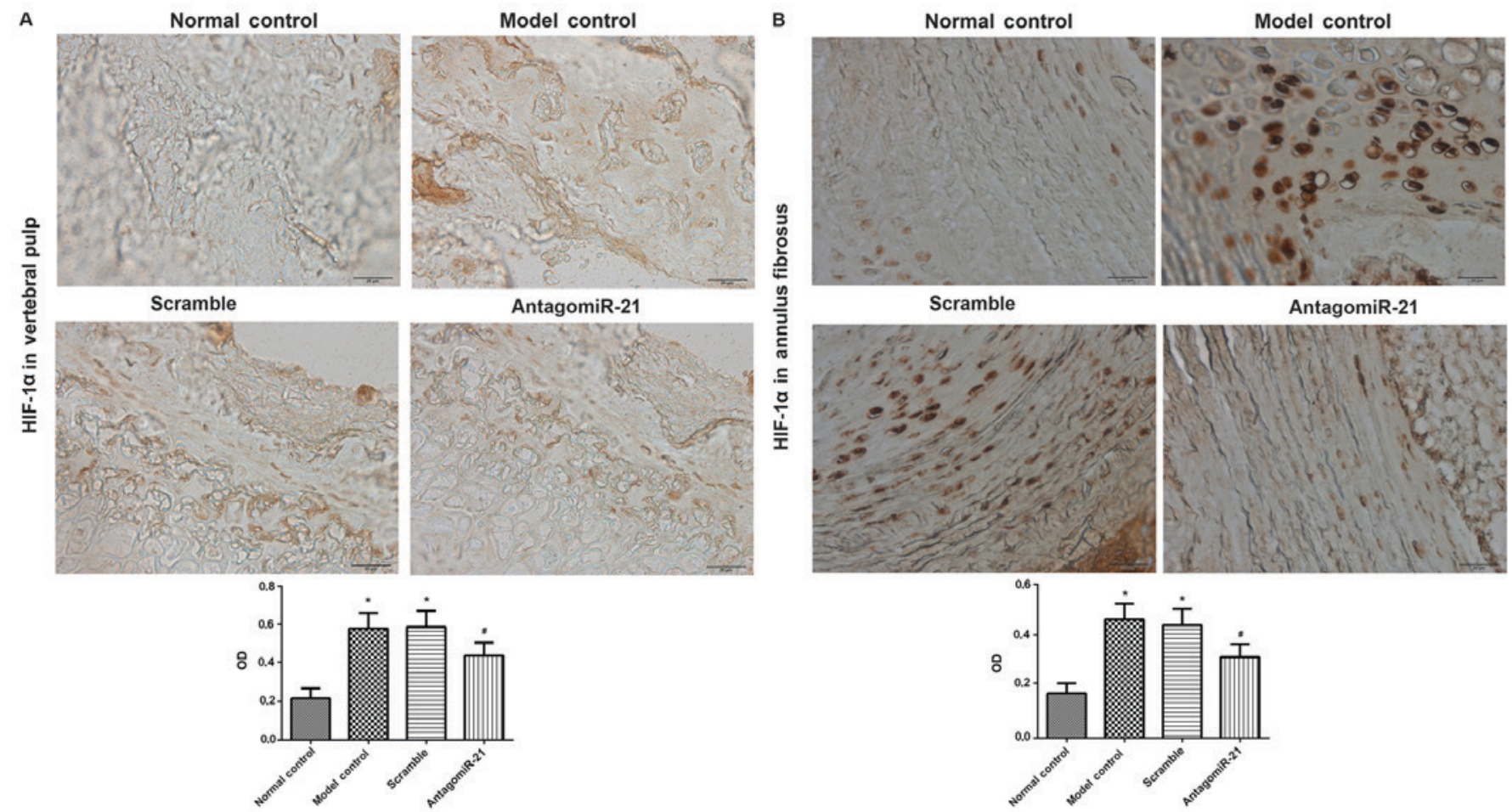

Figure 1. Vertebral pulp and annulus fibrosus was isolated from each group for the immunohistochemical analysis of HIF-1 $\alpha$ expression. AntagomiR-21 treatment decreased the expression of HIF- $1 \alpha$ in the (A) vertebral pulp and (B) annulus fibrosus. The quantification of immunohistochemistry results is presented below the representative images. $\mathrm{n}=15$ in each group. Scale bar, $20 \mu \mathrm{m}$. " $\mathrm{P}<0.05$ vs. normal control, "P<0.05 vs. scramble. HIF-1 $\alpha$, hypoxia inducible factor $1 \alpha$; antagomiR-21, microRNA-21 inhibitor; OD, optical density.

examine the effects of miR-21 on HIF-1 $\alpha$ and VEGF expression. The proteoglycan content in the lumbar spines and disc cell apoptosis was also detected.

\section{Materials and methods}

Animals. A total of 60 1-year-old specific pathogen free female Sprague-Dawley rats (150-200 g) were used in strict accordance with the guidelines for the Care and Use of Laboratory Animals (17). The present study was approved by the Animal Ethics Association of The Affiliated Second Hospital of Soochow University (Suzhou, China). The rats were purchased from the Animal Laboratory of the Academy of Medical Sciences (Beijing, China). They were kept in separate cages with free access to food and water, and a 12/12 h light/dark cycle (temperature, $25 \pm 1^{\circ} \mathrm{C}$; humidity, $50 \%$ ).

Experimental groups. Rats were randomly divided into four groups of 15. The normal control group received a skin incision, which were subsequently sutured. The rats in the three other groups underwent a previously described surgical procedure to induce the development of lumbar intervertebral disc degeneration (18). Briefly, the rats were anesthetized by an intraperitoneal injection of $350 \mathrm{mg} / \mathrm{kg} 6.5 \%$ chloral hydrate. The sacrospinal muscles, spinous processes, supraspinous ligaments, interspinous ligaments and posterolateral halves of the bilateral zygapophysial joints of the lumbar spine were removed.

Subsequently, rats in the model (control) group received a tail vein injection of normal saline; rats in the scramble group received a tail vein injection of $80 \mathrm{mg} / \mathrm{kg} /$ day control oligonucleotides (Guangzhou RiboBio Co., Ltd., Guangzhou, China); rats in the antagomiR-21 group received a tail vein injection of $80 \mathrm{mg} / \mathrm{kg} / \mathrm{day}$ of antagomiR-21 (Guangzhou RiboBio Co., Ltd.). Injections were administered for 8 weeks. Following this, rats were euthanized by an intraperitoneal overdose of pentobarbital sodium. Lumbar spines, including the L4 to L6 discs, were removed en bloc; the paravertebral muscles and the posterior columns were fully removed. The vertebral pulp and annulus fibrosus were isolated for immunohistochemical analysis of HIF-1 $\alpha$ and VEGF expression.

Immunohistochemical analysis. The metaphysis of the vertebral pulp and annulus fibrosus specimens were fixed in a $4 \%$ paraformaldehyde solution at room temperature for 30 min following threes washes with PBS. Next, tissues were dehydrated with a graded series of ethanol, infiltrated with xylene, and then embedded in paraffin before being cut into $6-\mu \mathrm{m}$-thick sections. The slides were then deparaffinization and rehydration with a graded ethanol series. Following this, the sections were depleted of endogenous peroxidase activity through the addition of methanolic $\mathrm{H}_{2} \mathrm{O}_{2}$ for $15 \mathrm{~min}$ and blocked with $10 \%$ normal goat serum (Abcam, Cambridge, MA, USA) at $37^{\circ} \mathrm{C}$ for $30 \mathrm{~min}$. The samples were incubated overnight at $4^{\circ} \mathrm{C}$ with anti-HIF-1 $\alpha$ (cat. no. ab113642; 1:200; Abcam) and anti-VEGFA (cat. no. ab46154; 1:100; Abcam). VEGFA is a common variant of VEGF, generally referred to as VEGF. The samples were subsequently incubated with a biotinylated rabbit secondary antibody (cat. no. BA1100; 1:375; Vector Laboratories, Inc., Burlingame, CA, USA) at $37^{\circ} \mathrm{C}$ for $1 \mathrm{~h}$. The bound secondary antibody was amplified using the Elite ABC kit (Vector Laboratories, Inc., Burlingame, CA, 

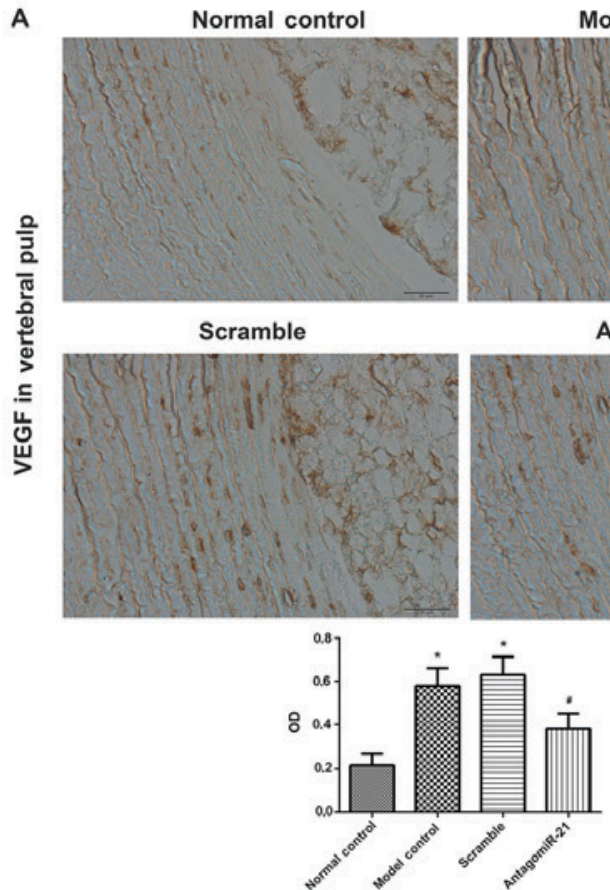

Model control

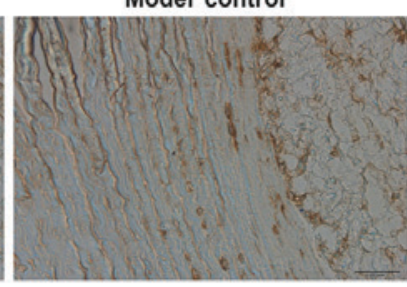

AntagomiR-21

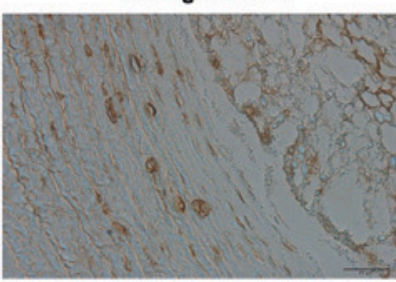

B
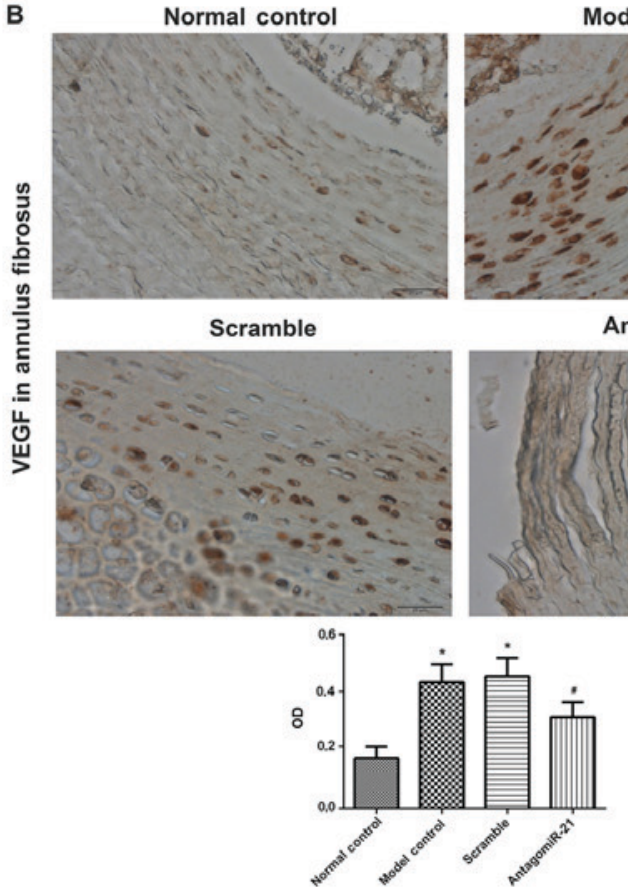

Model control

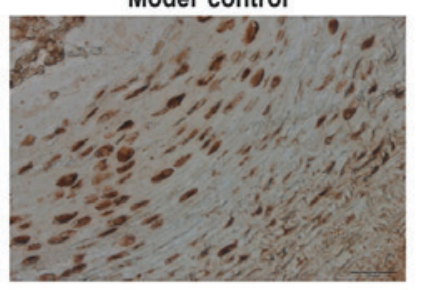

AntagomiR-21

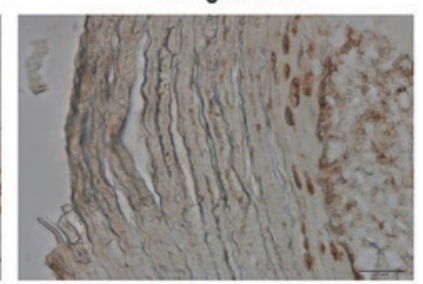

Figure 2. Vertebral pulp and annulus fibrosus was isolated from each group for immunohistochemical analysis of VEGF expression. AntagomiR-21 treatment decreased the expression of VEGF in the (A) vertebral pulp and the (B) annulus fibrosus. The quantification of immunohistochemistry results is presented below the representative images. $\mathrm{n}=15$ in each group. Scale bar, $20 \mu \mathrm{m}$. ${ }^{*} \mathrm{P}<0.05$ vs. normal control, ${ }^{*} \mathrm{P}<0.05$ vs. scramble. VEGF, vascular endothelial growth factor; antagomiR-21, microRNA-21 inhibitor; OD, optical density.

USA). The antibody-biotin-avidin-peroxidase complex was visualized using $0.02 \%$ 3,3'-diaminobenzidine. The sections were mounted onto gelatin-coated slides, air-dried overnight at room temperature and the coverslips were mounted using Permount medium (Thermo Fisher Scientific, Inc., Waltham, MA, USA). The slides were viewed using a light microscope (Olympus BH-2; Olympus Corporation, Tokyo, Japan; magnification, x400) and the optical density (OD) was analyzed by Image Pro Plus Version 6.0 image analyzing system (Media Cybernetics, Inc., Rockville, MD, USA).

Detection of proteoglycan content. The proteoglycan content in the lumbar spines was detected using the phloroglucinol method, as previously described (19). Briefly, $0.6 \mathrm{~g}$ tissue was

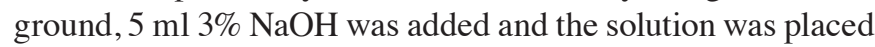
in a thermostatic oscillator at $40^{\circ} \mathrm{C}$ for $3 \mathrm{~h}$. Trypsin $(5 \mathrm{ml})$ was subsequently added for $2 \mathrm{~h}$ at $37^{\circ} \mathrm{C}$. The saccharide standard concentration was formulated according to a Phloroglucinol solution (Shanghai Macklin Biochemical Co., Ltd., Shanghai, China) following the manufacturer's protocol. Phloroglucinol solution $(5 \mathrm{ml})$ was added and the sample was placed in a water bath at $100^{\circ} \mathrm{C}$ for $8 \mathrm{~min}$. The absorbance of the solution was determined at $554 \mathrm{~nm}$ with a UV-visible spectrophotometer (WFZ-UV 2800H; Unico, Shanghai, China). Distilled water was used as a blank sample; the standard tube A value was determined and a standard curve was drawn. The proteoglycan content $(\mathrm{mg} / \mathrm{g})$ in the lumbar intervertebral disc tissue was calculated relative to the value of $\mathrm{A}$.

Apoptosis detection by terminal deoxynucleotidyl-transferase-mediated dUTP nick end labeling (TUNEL) staining. Lumbar spines were fixed in $4 \%$ paraformaldehyde

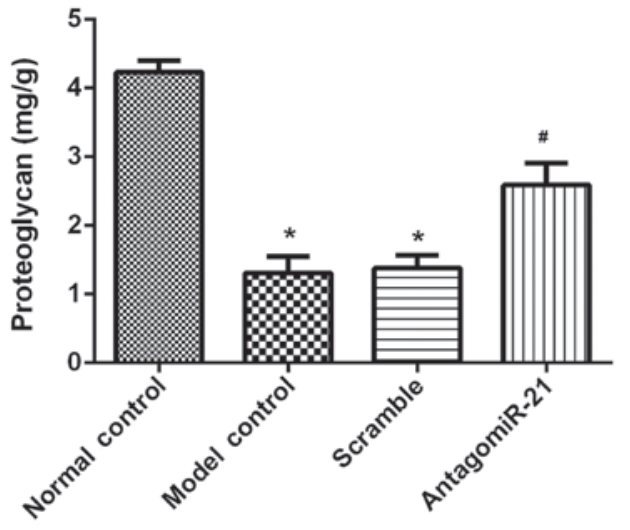

Figure 3. AntagomiR-21 treatment increased proteoglycan content in the lumbar spine. The lumbar spine proteoglycan content of each group was tested using the phloroglucinol method. $\mathrm{n}=15$ in each group. ${ }^{*} \mathrm{P}<0.05$ vs. normal control, ${ }^{\#} \mathrm{P}<0.05$ vs. scramble. AntagomiR-21, microRNA-21 inhibitor.

at $4^{\circ} \mathrm{C}$ for $48 \mathrm{~h}$, decalcified at $4^{\circ} \mathrm{C}$ in $20 \%$ EDTA for 5-7 weeks, embedded in paraffin and cut into $4-\mu \mathrm{m}$ thick sections along the midsagittal plane. An in situ TUNEL reaction was performed on two serial sections using the MK1020 apoptosis detection kit (Wuhan Boster Biological Technology, Ltd., Wuhan, China), according to the manufacturer's protocol. Apoptotic cells were imaged under a light microscope (magnification, x400). A total of 10 random fields were selected and the number of TUNEL-positive disc cells were compared with the total number of disc cells and expressed as a percentage.

Statistical analysis. Statistical analysis was performed with one-way analysis of variance followed by Tukey's test, using 


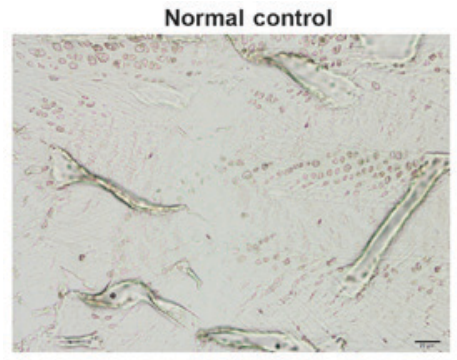

Scramble
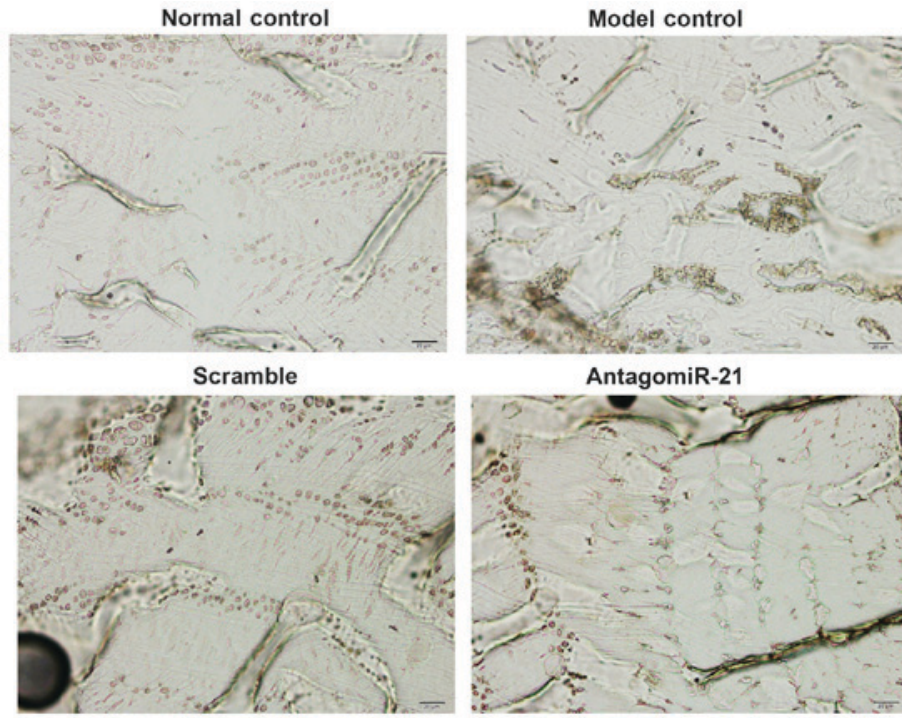

AntagomiR-21

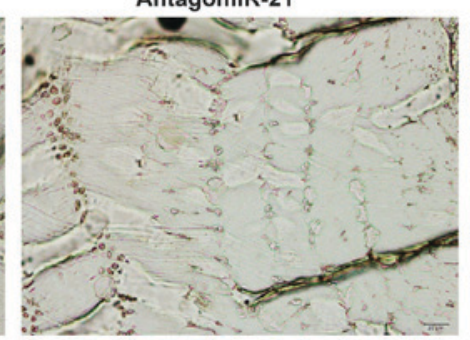

Figure 4. AntagomiR-21 treatment inhibited cell apoptosis in lumbar spines. The apoptosis rate in the lumbar spines of each group was detected with TUNEL staining. $\mathrm{n}=15$ in each group. Scale bar, $20 \mu \mathrm{m}$. ${ }^{*} \mathrm{P}<0.05$ vs. normal control, ${ }^{\sharp} \mathrm{P}<0.05$ vs. scramble. AntagomiR-21, microRNA-21 inhibitor; TUNEL, terminal deoxynucleotidyl-transferase-mediated dUTP nick end labeling.

SPSS 11.5 software (SPSS, Inc., Chicago, IL, USA). The data are presented as the mean \pm standard deviation from three independent experiments. Experiments were performed in triplicate. $\mathrm{P}<0.05$ was considered to indicate a statistically significant difference.

\section{Results}

AntagomiR-21 treatment decreased the expression of HIF-1a in the vertebral pulp and annulus fibrosus. The vertebral pulp and annulus fibrosus from rats in each group were isolated for immunohistochemical analysis of HIF-1 $\alpha$ expression. In the model and scramble groups, positive staining for HIF-1a (brown and yellow) was observed in the nucleus and cytoplasm (Fig. 1). The model and scramble groups exhibited significantly increased expression of HIF- $1 \alpha$ in the vertebral pulp and annulus fibrosus compared with the control group. Compared with the scramble group, antagomiR-21 treatment significantly decreased HIF-1 $\alpha$ expression in the vertebral pulp and annulus fibrosus.

AntagomiR-21 treatment decreased VEGF expression in the vertebral pulp and annulus fibrosus. The vertebral pulp and annulus fibrosus were also isolated for the immunohistochemical analysis of VEGF expression. Positive staining for the expression of VEGF (brown and yellow) was predominantly observed in the cytoplasm. Similar to the expression pattern of HIF-1 $\alpha$, VEGF expression in the model and scramble groups was significantly increased compared with the control group and VEGF expression was significantly decreased in the antagomiR-21 treatment group compared with the scramble group (Fig. 2).

AntagomiR-21 treatment increased the lumbar spine proteoglycan content. The phloroglucinol method was used to evaluate the effect of antagomiR-21 on the lumbar spine proteoglycan content. The results revealed that the proteoglycan content of the lumbar spine was significantly decreased in the model group compared with the control group. AntagomiR-21 treatment significantly increased the proteoglycan content in lumbar spines compared with the scramble group (Fig. 3).

AntagomiR-21 treatment inhibited cell apoptosis in lumbar spines. The effects of antagomiR-21 treatment on cell apoptosis in lumbar spines were investigated. The number of TUNEL-positive cells (brown) in the model group was significantly increased compared with the control group (Fig. 4). As expected, antagomiR-21 treatment significantly decreased the number of TUNEL-positive cells compared with the scramble group.

\section{Discussion}

In the present study, antagomiR-21 treatment was demonstrated to decrease the expression of HIF- $1 \alpha$ and VEGF in the vertebral pulp and annulus fibrosus of a rat model of intervertebral disc degeneration. Nucleus pulposus cell death mediated through apoptosis is involved in extracellular matrix degradation, which is a deleterious consequence of intervertebral disc degeneration (20). Liu et al (21) reported that miR-21 is upregulated in degenerative human nucleus pulposus tissues compared with normal tissues. Furthermore, Liu et al (21) demonstrated that miR-21 administration promotes nucleus pulposus cell proliferation.

To the best of our knowledge, the present study provided novel evidence to demonstrate the role of miR-21 in the regulation of angiogenesis, as evidenced by the decreased expression of HIF-1 $\alpha$ and VEGF following antagomiR-21 treatment in a rat model of intervertebral disc degeneration. Consistent with these findings, Liu et al (14) reported that miR-21 overexpression increases HIF-1 $\alpha$ and VEGF expression and induces tumor angiogenesis. Zhao et al (22) suggested that inhibition of angiogenesis with antagomiR-21 occurs through the HIF-1 $\alpha /$ VEGF/VEGF receptor 2 signaling pathway. 
HIF-1 is a key transcription factor expressed in response to hypoxic stress and is closely associated with angiogenesis. HIF-1 is a heterodimeric transcription factor containing HIF-1 $\alpha$ and HIF-1 $\beta$ subunits (23). Under hypoxic conditions, HIF-1 $\alpha$ accumulates in the cytoplasm and subsequently translocates into the nucleus. HIF-1 $\alpha$ and HIF- $1 \beta$ can then dimerize and bind to hypoxia response elements to stimulate the transcription of a large number of genes, including prostaglandin synthase, angiopoietin, protein tyrosine phosphatase, erythropoietin and VEGF (24,25). Zhu et al (26) suggested that mutual promotion of HIF-1 $\alpha$ expression occurs during the process of lumbar intervertebral disc degeneration and that the expression of HIF- $1 \alpha$ is significantly associated with microvessel density, which provides evidence for the association between HIF-1 $\alpha$ expression and angiogenesis in lumbar intervertebral disc degeneration of rats.

The present study also revealed that antagomiR-21 treatment increased proteoglycan content and inhibited cell apoptosis in lumbar spines. A decrease in proteoglycan content is consistently detected with degeneration, particularly in the center of the disc (27). The induction of disc cell apoptosis is closely associated with intervertebral disc degeneration (28). Thus, it was concluded that antagomiR-21 treatment exerted a protective role in the rat model of intervertebral disc degeneration. However, the present study only discussed the effect of antagomiR-21 treatment on expression of HIF-1 $\alpha$ and VEGF in the vertebral pulp, annulus fibrosus, lumbar spine proteoglycan content, and lumbar spine cell apoptosis of a rat model of intervertebral disc degeneration. The association of an antagomiR-21-mediated decrease in the expression of HIF-1 $\alpha$ and VEGF with proteoglycan content and/or cell apoptosis in lumbar spines was not fully elucidated, which was the limitation of the present study. In addition, the underlying mechanism by which antagomiR-21 treatment decreased expression of HIF-1 $\alpha$ and VEGF also requires further investigation.

In conclusion, the present study demonstrated that antagomiR-21 treatment exerted a protective role in the rat model of intervertebral disc degeneration by increasing the proteoglycan content and inhibiting cell apoptosis, at least in part through HIF-1 $\alpha$ and VEGF expression regulation. The findings of the current study demonstrate that antagomiR-21 may be a novel approach for the treatment of intervertebral disc degeneration.

\section{Acknowledgements}

Not applicable.

\section{Funding}

The present research was supported by the National Natural Science Foundation of China (grant no. 81572179), the Jiangsu Provincial Grant (grant no. BL 2014044), the pre-research project of the Affiliated Second Hospital (grant no. SDFEYQN1608) and the Jiangsu Province's Young Medical Talents Program (grant no. QNRC2016880).

\section{Availability of data and materials}

The datasets used and/or analyzed during the current study are available from the corresponding author on reasonable request.

\section{Authors' contributions}

XS and YX conceived and designed the study. XS, QG, and JY performed the experiments. XS and QG wrote the paper. YX reviewed and edited the manuscript. All authors read and approved the manuscript.

\section{Ethics approval and consent to participate}

The present study was approved by the Animal Ethics Association of The Affiliated Second Hospital of Soochow University (Suzhou, China).

\section{Consent for publication}

Not applicable.

\section{Competing interests}

The authors declare that they have no competing interests.

\section{References}

1. Kang JD, Stefanovic-Racic M, Mcintyre LA, Georgescu HI and Evans $\mathrm{CH}$ : Toward a biochemical understanding of human intervertebral disc degeneration and herniation. Contributions of nitric oxide, interleukins, prostaglandin E2, and matrix metalloproteinases. Spine (Phila Pa 1976) 22: 1065-1073, 1997.

2. Luoma K, Riihimäki H, Luukkonen R, Raininko R, Viikari-Juntura E and Lamminen A: Low back pain in relation to lumbar disc degeneration. Spine (Phila Pa 1976) 25: 487-492, 2000.

3. Freemont AJ, Watkins A, Le Maitre C, Jeziorska M and Hoyland JA: Current understanding of cellular and molecular events in intervertebral disc degeneration: Implications for therapy. J Pathol 196: 374-379, 2002.

4. Fairbank J: Clinical importance of the intervertebral disc, or back pain for biochemists. Biochem Soc Trans 30: 829-831, 2002.

5. Adams MA and Roughley PJ: What is intervertebral disc degeneration, and what causes it? Spine (Phila Pa 1976) 31: 2151-2161, 2006.

6. Binch ALA, Phillips KL, Chiverton N, Cole A, Michael AR, Breakwell L, Cross AK, Le Maitre CL: Role of semaphorins in angiogenesis and innervation in human intervertebral disc degeneration. Global Spine Journal 04: 1834-1841, 2014.

7. Ali R, Le-Maitre CL, Richardson SM, Hoyland JA and Freemont AJ: Connective tissue growth factor expression in human intervertebral disc: Implications for angiogenesis in intervertebral disc degeneration. Biotech Histochem 83: 239-245, 2008.

8. Lee JM, Song JY, Baek M, Jung HY, Kang H, Han IB, Kwon YD and Shin DE: Interleukin-1 $\beta$ induces angiogenesis and innervation in human intervertebral disc degeneration. J Orthop Res 29: 265-269, 2011.

9. Wang J, Chen H, Yuan W, Cao P, Shi L, Li R and Zang F: Analysis on angiogenesis in degenerative intervertebral disc and relevant factors. Zhonghua Guke Zazhi 35: 1200-1205, 2015 (In Chinese).

10. David G, Ciurea AV, Iencean SM and Mohan A: Angiogenesis in the degeneration of the lumbar intervertebral disc. J Med Life 3: 154-161, 2010.

11. Calin GA and Croce CM: MicroRNA signatures in human cancers. Nat Rev Cancer 6: 857-866, 2006.

12. Zhao Y, Xu Y, Luo F, Xu W, Wang B, Pang Y, Zhou J, Wang X and Liu Q: Angiogenesis, mediated by miR-21, is involved arsenite-induced carcinogenesis. Toxicol Lett 223: 35-41, 2013.

13. Sabatel C, Malvaux L, Bovy N, Deroanne C, Lambert V, Gonzalez ML, Colige A, Rakic JM, Noël A, Martial JA and Struman I: MicroRNA-21 exhibits antiangiogenic function by targeting RhoB expression in endothelial cells. PLoS One 6: e16979, 2011.

14. Liu LZ, Li C, Chen Q, Jing Y, Carpenter R, Jiang Y, Kung HF, Lai L and Jiang BH: MiR-21 induced angiogenesis through AKT and ERK activation and HIF-1 $\alpha$ expression. PLoS One 6: e19139, 2011. 
15. Chen X, Liu J, He B, Li Y, Liu S, Wu B, Wang S, Zhang S, $\mathrm{Xu} X$ and Wang J: Vascular endothelial growth factor (VEGF) regulation by hypoxia inducible factor-1 alpha (HIF1A) starts and peaks during endometrial breakdown, not repair, in a mouse menstrual-like model. Hum Reprod 30: 2160-2170, 2015.

16. Wu WJ, Zhang XK, Zheng XF, Yang YH, Jiang SD and Jiang LS: SHH-dependent knockout of HIF-1 alpha accelerates the degenerative process in mouse intervertebral disc. Int J Immunopathol Pharmacol 26: 601-609, 2013.

17. Bayne K: Revised guide for the care and use of laboratory animals available. american physiological society. Physiologist 39: 199, 208-111, 1996.

18. Zhao CQ, Zhang YH, Jiang SD, Jiang LS and Dai LY: Both endoplasmic reticulum and mitochondria are involved in disc cell apoptosis and intervertebral disc degeneration in rats. Age (Dordr) 32: 161-177, 2010

19. Wu B, Meng C, Wang H, Jia C and Zhao Y: Proteoglycan and collagen type II in the adjacent intervertebral disc of the cervical instability models. Zhongguo Zuzhi Gongcheng Yanjiu 17: 5421-5426, 2013 (In Chinese).

20. Wei A, Brisby H, Chung SA and Diwan AD: Bone morphogenetic protein-7 protects human intervertebral disc cells in vitro from apoptosis. Spine J 8: 466-474, 2008.

21. Liu H, Huang X, Liu X, Xiao S, Zhang Y, Xiang T, Shen X, Wang $G$ and Sheng B: miR-21 promotes human nucleus pulposus cell proliferation through PTEN/AKT signaling. Int J Mol Sci 15: 4007-4018, 2014.

22. Zhao D, Tu Y, Wan L, Bu L, Huang T, Sun X, Wang K and Shen B: In vivo monitoring of angiogenesis inhibition via down-regulation of mir-21 in a VEGFR2-luc murine breast cancer model using bioluminescent imaging. PLoS One 8: e71472, 2013.
23. Semenza GL: Vascular responses to hypoxia and ischemia. Arterioscler Thromb Vasc Biol 30: 648-652, 2010.

24. Wang GL, Jiang BH, Rue EA and Semenza GL: Hypoxia-inducible factor 1 is a basic-helix-loop-helix-PAS heterodimer regulated by cellular O2 tension. Proc Natl Acad Sci USA 92: 5510-5514, 1995.

25. Rey S and Semenza GL: Hypoxia-inducible factor-1-dependent mechanisms of vascularization and vascular remodelling. Cardiovasc Res 86: 236-242, 2010.

26. Zhu L, Ye W and Cao Y: The relationship between the expression of cyclooxygenase-2, hypoxia-inducible factor- $1 \alpha$ and angiogenesis in lumbar intervertebral disc degeneration of rats. Jiang Yaotong Zazhi 33: 174-178, 2012 (In Chinese).

27. Lyons G, Eisenstein SM and Sweet MB: Biochemical changes in intervertebral disc degeneration. Biochim Biophys Acta 673: 443-453, 1981.

28. Kohyama K, Saura R, Doita M and Mizuno K: Intervertebral disc cell apoptosis by nitric oxide: Biological understanding of intervertebral disc degeneration. Kobe J Med Sci 46: 283-295, 2000.

This work is licensed under a Creative Commons Attribution-NonCommercial-NoDerivatives 4.0 International (CC BY-NC-ND 4.0) License. 